Supporting Information

\title{
Effect of Nanogap Curvature on SERS: A Finite-Difference Time-Domain Study
}

\author{
Daedu Lee and Sangwoon Yoon* \\ Department of Chemistry, Dankook University \\ 152 Jukjeon-ro, Suji-gu, Yongin, Gyeonggi 448-701, Korea \\ *E-mail: sangwoon@dankook.ac.kr
}




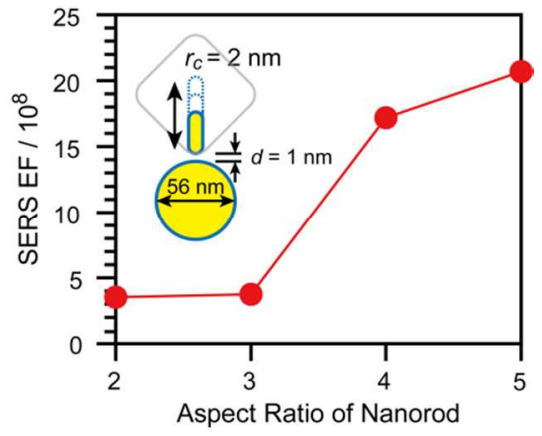

Figure S1. SERS EF of Au nanorod-nanosphere dimers when the aspect ratio of the nanorod increases from 2 to 5 . The curvature radius of the end of the nanorod $\left(r_{c}\right)$ and the gap distance $(d)$ were fixed at 2 and $1 \mathrm{~nm}$, respectively. 